\title{
Stability of cylindrical thin shell wormhole during evolution of universe from inflation to late time acceleration
}

\author{
M.R. Setare ${ }^{a}$ and A. Sepehri ${ }^{b}$ \\ ${ }^{a}$ Department of Science, Campus of Bijar, University of Kurdistan, \\ Bijar, Iran \\ ${ }^{b}$ Faculty of Physics, Shahid Bahonar University, \\ P.O. Box 76175, Kerman, Iran \\ E-mail: rezakord@ipm.ir, alireza.sepehri@uk.ac.ir
}

ABSTRACT: In this paper, we consider the stability of cylindrical wormholes during evolution of universe from inflation to late time acceleration epochs. We show that there are two types of cylindrical wormholes. The first type is produced at the corresponding point where $\mathrm{k}$ black F-strings are transited to BIon configuration. This wormhole transfers energy from extra dimensions into our universe, causes inflation, loses it's energy and vanishes. The second type of cylindrical wormhole is created by a tachyonic potential and causes a new phase of acceleration. We show that wormhole parameters grow faster than the scale factor in this era, overtake it at ripping time and lead to the destruction of universe at big rip singularity.

KEywords: Strings and branes phenomenology, Phenomenology of Large extra dimensions

ARXIV EPRINT: 1412.8666 


\section{Contents}

1 Introduction 1

2 The birth and death of cylindrical wormholes during inflation 2

3 The birth and death of cylindrical wormhole during late time acceleration

\section{Introduction}

The idea of spacetime wormhole was introduced in 1930s by Einstein and Rosen [1] and then extended and popularized by Morris, Thorne [2] and Visser [3]. One of important types of these objects that may help us learn more about extra dimensions are thin shell wormholes (TSW). These objects are constructed by Visser from an energy-momentum at the throat through cut-and-paste technique satisfying the proper junction conditions. Visser proposed the first construction of thin shell wormholes in a 3+1-dimensional flat Minkowski space-time [3] and then extended it to the Schwarzschild spacetime [4, 5].

Now, the main question arises what is the origin of these wormholes in different epochs of universe? We answer to this question in BIonic model [6-10] which allows us to take into account the wormhole in addition to tachyons in brane-antibrane system. In this model, at the corresponding point where $\mathrm{k}$ black fundamental strings are transited to BIon, two universe-branes and one thin shell wormhole are born. This wormhole is a channel for flowing energy from anti-universe-brane to our universe and has the main role in making the inflation. After a short period of time, wormhole loses it's energy, vanishes, inflation ends and deceleration era begins. With decreasing separation between universe branes, tachyon is created and causes formation of the second type of unstable thin shell wormholes, named as tachyonic TSWs. At this stage, universe evolved from a non-phantom deceleration era to a phantom acceleration phase and ends up in a big rip singularity.

To construct expansion history of universe in BIon, we make use of thin shell wormholes that have a cylindrical symmetry. Until now, less discussions have been made on cylindrically symmetric thin shell wormholes [11-18]. For example, some authors presented a model for the dynamics of non rotating cylindrical thin-shell wormholes. They calculated the time evolution of the throat of special type of wormhole whose metric has the form associated to local cosmic strings [11, 12]. Also, they extended their calculations and built this type of wormholes within the framework of the Brans Dicke scalar-tensor theory of gravity [13]. Some other authors constructed cylindrical, traversable wormholes by gluing 
two copies of a cosmic strings manifold with a positive cosmological constant [14]. In another scenario, authors showed that the existence of wormholes with cylindrical topology does not require violation of the weak or null energy conditions near the throat $[15,16]$. Some other investigators discussed that the wormhole and the cosmic string geometries are locally indistinguishable, although, their global properties are different [17]. In a recent work, researchers investigated the stability of cylindrical thin shell wormholes in parallel to the method used in spherically symmetric thin shell wormholes [18]. We will extend these calculations to string theory and will show that these wormholes are unstable both with the main causes of inflation and with a late time acceleration.

The outline of the paper is as follows. In section 2, we will consider the evolution of cylindrical thin shell wormholes and their annihilation during the inflation era of universe. In section 3, we will introduce a new type of cylindrical thin shell wormhole, named as tachyonic TSWs. These objects were born due to a tachyonic potential between braneantibranes. The last section is devoted to summary and conclusion.

\section{The birth and death of cylindrical wormholes during inflation}

In this section, we will show that cylindrical wormholes are born at the beginning of inflation and vanish at the end of this epoch. We obtain the location of throat in terms of BIonic parameters.

First let us consider a general cylindrical metric which is given by [12]:

$$
d s^{2}=B(r)\left(-d t^{2}+d r^{2}\right)+C(r) d \phi^{2}+D(r) d z^{2}
$$

in which $\mathrm{B}(\mathrm{r}), \mathrm{C}(\mathrm{r})$ and $\mathrm{D}(\mathrm{r})$ are as a function of $\mathrm{r}$ only and $\mathrm{r}=\mathrm{V}(\mathrm{t})$ is the location of the throat. The standard energy momentum on the shell is:

$$
\begin{aligned}
\rho & =-\left(\frac{D^{\prime}}{D}+\frac{C^{\prime}}{C}\right) \sqrt{\Delta} \\
p_{z} & =\frac{1}{\sqrt{\Delta}}\left[2 \ddot{V}+2 \frac{B^{\prime}}{B} \dot{V}^{2}+\frac{B^{\prime}}{B^{2}}+\frac{C^{\prime}}{C} \Delta\right], \\
p_{\phi} & =\frac{1}{\sqrt{\Delta}}\left[2 \ddot{V}+2 \frac{B^{\prime}}{B} \dot{V}^{2}+\frac{B^{\prime}}{B^{2}}+\frac{D^{\prime}}{D} \Delta\right]
\end{aligned}
$$

where $\Delta=\frac{1}{B}+\dot{V}^{2}$. Now, we discuss the origin of wormhole in cosmic space. In our model, coincided with the birth of universe, wormhole is born at corresponding point where the thermodynamics of $\mathrm{k}$ non-extremal black fundamental strings has been matched to that of the BIon configuration. The supergravity solution for $\mathrm{k}$ coincident non-extremal black $\mathrm{F}$-strings lying along the $\mathrm{z}$ direction is:

$$
\begin{array}{rlrl}
d s^{2} & =H^{-1}\left(-f d t^{2}+d z^{2}\right)+f^{-1} d r^{2}+r^{2} d \Omega_{7}^{2} \\
e^{2 \phi} & =H^{-1}, & B_{0} & =H^{-1}-1, \\
H & =1+\frac{r_{0}^{6} \sinh ^{2} \alpha}{r^{6}}, & f=1-\frac{r_{0}^{6}}{r^{6}} .
\end{array}
$$


From this metric, the mass density along the $\mathrm{z}$ direction can be found $[7,8]$ :

$$
\frac{d M_{F 1}}{d z}=T_{F 1} k+\frac{16\left(T_{F 1} k \pi\right)^{3 / 2} T^{3}}{81 T_{D 3}}+\frac{40 T_{F 1}^{2} k^{2} \pi^{3} T^{6}}{729 T_{D 3}^{2}} .
$$

On the other hand, for finite temperature BIon, the metric is [6]:

$$
d s^{2}=-d t^{2}+d r^{2}+r^{2}\left(d \theta^{2}+\sin ^{2} \theta d \phi^{2}\right)+\sum_{i=1}^{6} d x_{i}^{2} .
$$

Choosing the world volume coordinates of the D3-brane as $\left\{\sigma^{a}, a=0 \ldots 3\right\}$ and defining $\tau=\sigma^{0}, \sigma=\sigma^{1}$, the coordinates of BIon are given by $[6-8]$ :

$$
t\left(\sigma^{a}\right)=\tau, r\left(\sigma^{a}\right)=\sigma, \quad x_{1}\left(\sigma^{a}\right)=z(\sigma), \quad \theta\left(\sigma^{a}\right)=\sigma^{2}, \quad \phi\left(\sigma^{a}\right)=\sigma^{3}
$$

and the remaining coordinates $x_{i=2, \ldots 6}$ are constant. The embedding function $z(\sigma)$ describes the bending of the brane. Let $\mathrm{z}$ be a transverse coordinate to the branes and $\sigma$ be the radius on the world-volume. The induced metric on the brane is then:

$$
\gamma_{a b} d \sigma^{a} d \sigma^{b}=-d \tau^{2}+\left(1+z^{\prime}(\sigma)^{2}\right) d \sigma^{2}+\sigma^{2}\left(d \theta^{2}+\sin ^{2} \theta d \phi^{2}\right)
$$

so that the spatial volume element is $d V_{3}=\sqrt{1+z^{\prime}(\sigma)^{2}} \sigma^{2} d \Omega_{2}$. We impose the two boundary conditions $z(\sigma) \rightarrow 0$ for $\sigma \rightarrow \infty$ and $z^{\prime}(\sigma) \rightarrow-\infty$ for $\sigma \rightarrow \sigma_{0}$, where $\sigma_{0}$ is the minimal two-sphere radius of the configuration. For this BIon, the mass density along the $\mathrm{z}$ direction can be obtained $[7,8]$ :

$$
\frac{d M_{\mathrm{BIon}}}{d z}=T_{F 1} k+\frac{3 \pi T_{F 1}^{2} k^{2} T^{4}}{32 T_{D 3}^{2} \sigma_{0}^{2}}+\frac{7 \pi^{2} T_{F 1}^{3} k^{3} T^{8}}{512 T_{D 3}^{4} \sigma_{0}^{4}} .
$$

Comparing the mass densities for BIon to the mass density for the F-strings, we see that the thermal BIon configuration behaves like k F-strings at $\sigma=\sigma_{0}$. At this corresponding point, $\sigma_{0}$ should have the following dependence on the temperature:

$$
\sigma_{0}=\left(\frac{\sqrt{k T_{F 1}}}{T_{D 3}}\right)^{1 / 2} \sqrt{T}\left[C_{0}+C_{1} \frac{\sqrt{k T_{F 1}}}{T_{D 3}} T^{3}\right]
$$

where $T_{F 1}=4 k \pi^{2} T_{D 3} g_{s} l_{s}^{2}, C_{0}, C_{1}, F_{0}, F_{1}$ and $F_{2}$ are numerical coefficients which can be determined by requiring that $T^{3}$ and $T^{6}$ terms in eqs. (2.4) and (2.8) are consistent with each other. At this point, two universes and one wormhole have been born. The metric of these FRW universes is:

$$
d s_{\mathrm{Uni} 1}^{2}=d s_{\mathrm{Uni} 2}^{2}=-d t^{2}+a(t)^{2}\left(d x^{2}+d y^{2}+d z^{2}\right) .
$$

We assume that universes are located on D3-branes and don't have any contribution in mass density along z direction. For this reason, we write:

$$
p_{z}=\frac{d M_{F 1}}{d z} \rightarrow \frac{1}{\sqrt{\Delta}}\left[2 \ddot{V}+2 \frac{B^{\prime}}{B} \dot{V}^{2}+\frac{B^{\prime}}{B^{2}}+\frac{C^{\prime}}{C} \Delta\right]=T_{F 1} k+\frac{16\left(T_{F 1} k \pi\right)^{3 / 2} T^{3}}{81 T_{D 3}}+\frac{40 T_{F 1}^{2} k^{2} \pi^{3} T^{6}}{729 T_{D 3}^{2}} .
$$


Assuming $B^{\prime}\left(V_{0}\right)=C^{\prime}\left(V_{0}\right)=0$, we can solve the above equation:

$$
\begin{aligned}
V(t) & =\exp \left(\int P_{0} d t\right) \\
P_{0} & =T_{F 1} k+\frac{16\left(T_{F 1} k \pi\right)^{3 / 2} T^{3}}{81 T_{D 3}}+\frac{40 T_{F 1}^{2} k^{2} \pi^{3} T^{6}}{729 T_{D 3}^{2}} .
\end{aligned}
$$

Using this equation and equation (2.9) and assuming that throat of a stringy wormhole is equal to throat of a cylindrical wormhole $V_{0}=\sigma_{0}$, we can derive the universe temperature in terms of time:

$$
\begin{aligned}
V & =\sigma_{0} \rightarrow \\
\exp \left(\int P_{0} d t\right) & =\left(\frac{\sqrt{k T_{F 1}}}{T_{D 3}}\right)^{1 / 2} \sqrt{T}\left[C_{0}+C_{1} \frac{\sqrt{k T_{F 1}}}{T_{D 3}} T^{3}\right] \rightarrow \\
T^{-1} & \sim\left[\left(\frac{40 T_{F 1} k \pi^{3}}{729}\right)^{2 / 3} t^{2 / 3}+\left(\frac{40 T_{F 1} k \pi^{3}}{729}\right)^{2 / 11} t^{2 / 11}+\ln \left(\frac{16 T_{D 3}\left(T_{F 1} k \pi\right)^{1 / 2}}{81} t\right)\right], \\
V & =\sigma_{0}=0, \bar{C}_{0}=-C_{0} \rightarrow T_{\text {end }}=\frac{\bar{C}_{0} \sqrt{T_{D 3}}}{C_{1} k T_{F 1}} \rightarrow \\
t_{\text {end }} & \sim \frac{C_{1} k T_{F 1}}{\bar{C}_{0} \sqrt{T_{D 3}}}\left[\left(\frac{40 T_{F 1} k \pi^{3}}{729}\right)^{2 / 11}+\frac{16 T_{D 3}\left(T_{F 1} k \pi\right)^{1 / 2}}{81}\right]^{-1} .
\end{aligned}
$$

This equation shows that temperature was infinity at the beginning, decreased with time and tended to $T_{\text {end }}$ at large values of time $\left(t=t_{\text {end }}\right)$. Consequently, throat of a wormhole is decreased with time and tends to zero at $t=t_{\text {end }}$.

After that, wormhole transfers energy from extra dimensions into our universe and causes inflation. Simultaneously, one stringy wormhole is formed in BIon. To compare a stringy wormhole with a cylindrical wormhole, we will construct a stringy wormhole in BIon. Putting k units of F-string charge along the radial direction and using equation (2.7), we obtain $[6-8]$ :

$$
z(\sigma)=\int_{\sigma}^{\infty} d \hat{\sigma}\left(\frac{F(\hat{\sigma})^{2}}{F\left(\sigma_{0}\right)^{2}}-1\right)^{-\frac{1}{2}} .
$$

In finite temperature BIon $F(\sigma)$ is given by

$$
F(\sigma)=\sigma^{2} \frac{4 \cosh ^{2} \alpha-3}{\cosh ^{4} \alpha}
$$

where $\cosh \alpha$ is determined by the function:

$$
\cosh ^{2} \alpha=\frac{3}{2} \frac{\cos \frac{\delta}{3}+\sqrt{3} \sin \frac{\delta}{3}}{\cos \delta}
$$

with the definitions:

$$
\cos \delta \equiv \bar{T}^{4} \sqrt{1+\frac{k^{2}}{\sigma^{4}}}, \quad \bar{T} \equiv\left(\frac{9 \pi^{2} N}{4 \sqrt{3} T_{D_{3}}}\right) T, \quad \kappa \equiv \frac{k T_{F 1}}{4 \pi T_{D_{3}}} .
$$


In above equation, $\mathrm{T}$ is the finite temperature of BIon, $\mathrm{N}$ is the number of D3-branes and $T_{D_{3}}$ and $T_{F 1}$ are tensions of brane and fundamental strings respectively. Attaching a mirror solution to eq. (2.13), we construct a wormhole configuration. The separation distance $\bar{\Delta}=2 z\left(\sigma_{0}\right)$ between the N D3-branes and $\mathrm{N}$ anti D3-branes for a given brane-antibrane wormhole configuration is defined by the four parameters $\mathrm{N}, \mathrm{k}, \mathrm{T}$ and $\sigma_{0}$. We have:

$$
\bar{\Delta}=2 z\left(\sigma_{0}\right)=2 \int_{\sigma_{0}}^{\infty} d \hat{\sigma}\left(\frac{F(\dot{\sigma})^{2}}{F\left(\sigma_{0}\right)^{2}}-1\right)^{-\frac{1}{2}} .
$$

In the limit of small temperatures, we obtain:

$$
\bar{\Delta}=\frac{2 \sqrt{\pi} \Gamma(5 / 4)}{\Gamma(3 / 4)} \sigma_{0}\left(1+\frac{8}{27} \frac{k^{2}}{\sigma_{0}^{4}} \bar{T}^{8}\right) .
$$

Let us now discuss the early inflationary model of universe in thermal BIon. For this, we need to compute the contribution of the BIonic system with the four-dimensional universe energy momentum tensor. The EM tensor for one BIonic system with N D3-branes and $\mathrm{k}$ F-string charges is $[7,8]$,

$$
\begin{aligned}
T^{00} & =\frac{2 T_{D 3}^{2}}{\pi T^{4}} \frac{F(\sigma)}{\sqrt{F^{2}(\sigma)-F^{2}\left(\sigma_{0}\right)}} \sigma^{2} \frac{4 \cosh ^{2} \alpha+1}{\cosh ^{4} \alpha} \\
T^{i i} & =-\gamma^{i i} \frac{8 T_{D 3}^{2}}{\pi T^{4}} \frac{F(\sigma)}{\sqrt{F^{2}(\sigma)-F^{2}\left(\sigma_{0}\right)}} \sigma^{2} \frac{1}{\cosh ^{2} \alpha}, \quad i=1,2,3 \\
T^{44} & =\frac{2 T_{D 3}^{2}}{\pi T^{4}} \frac{F(\sigma)}{F\left(\sigma_{0}\right)} \sigma^{2} \frac{4 \cosh ^{2} \alpha+1}{\cosh ^{4} \alpha} .
\end{aligned}
$$

This equation shows that with increasing temperature in BIonic system, the energymomentum tensors decreases. This is due to the fact that when spikes of branes and antibranes are well separated, wormhole is not formed, so there isn't any channel for flowing energy from universe branes into extra dimensions and consequently the temperature is very high, however when two universe branes are close to each other and connected by a wormhole, the temperature is reduced to the lower values.

Also, in this model, we introduce two four dimensional universes that interact with each other via a wormhole and form a binary system. In this model, $\mathrm{z}$ is the transverse direction between two universes and only the wormhole has a momentum in this direction. To obtain the energy-momentum tensor in this system, we use the Einstein's field equation in presence of fluid flow that reads as:

$$
R_{i j}-\frac{1}{2} \mathrm{~g}_{i j} R=k T_{i j}
$$

Using this equation, we can obtain the energy momentum tensor for the universe-wormhole:

$$
\begin{aligned}
T^{00} & =6 \frac{\dot{a}^{2}}{a^{2}}+\left(\frac{D^{\prime}}{D}+\frac{C^{\prime}}{C}\right) \sqrt{\Delta} \\
T^{i i} & =4 \frac{\ddot{a}}{a}+2 \frac{\dot{a}^{2}}{a^{2}}+\frac{1}{\sqrt{\Delta}}\left[2 \ddot{V}+2 \frac{B^{\prime}}{B} \dot{V}^{2}+\frac{B^{\prime}}{B^{2}}+\frac{D^{\prime}}{D} \Delta\right], \quad i=1,2,3 \\
T^{44} & =\frac{1}{\sqrt{\Delta}}\left[2 \ddot{V}+2 \frac{B^{\prime}}{B} \dot{V}^{2}+\frac{B^{\prime}}{B^{2}}+\frac{C^{\prime}}{C} \Delta\right] .
\end{aligned}
$$


On the other hand, such a higher-dimensional stress-energy tensor is assumed to be that of a perfect fluid and of the form

$$
T_{i}^{j}=\operatorname{diag}[-p,-p,-p,-\bar{p},-p,-p,-p, \rho],
$$

where $\bar{p}$ is the pressure in the extra space-like (z) dimension. Using the energy momentum tensor of equations (2.20) and (2.22) in the conservation law of equations (2.21) and employing (2.23), we write:

$$
\begin{aligned}
\rho & =\rho_{\text {Uni } 1}+\rho_{\text {Uni } 2}+\rho_{\text {wormhole }}=\rho_{\text {BIon }} \\
p_{i, \text { tot }} & =p_{i, \text { Uni1 }}+p_{i, \text { Uni } 2}+p_{i, \text { wormhole }}=p_{i, \text { BIon }}, \mathrm{i}=1,2,3 \\
p_{z, \text { tot }} & =p_{z, \text { wormhole }}=p_{z, \text { BIon }} .
\end{aligned}
$$

By using equations (2.20), (2.22) and (2.24), we obtain:

$$
\begin{aligned}
6 \frac{\dot{a}^{2}}{a^{2}}+\left(\frac{D^{\prime}}{D}+\frac{C^{\prime}}{C}\right) \sqrt{\Delta} & =\frac{2 T_{D 3}^{2}}{\pi T^{4}} \frac{F(\sigma)}{\sqrt{F^{2}(\sigma)-F^{2}\left(\sigma_{0}\right)}} \sigma^{2} \frac{4 \cosh ^{2} \alpha+1}{\cosh ^{4} \alpha} \\
4 \frac{\ddot{a}}{a}+2 \frac{\dot{a}^{2}}{a^{2}}+\frac{1}{\sqrt{\Delta}}\left[2 \ddot{V}+2 \frac{B^{\prime}}{B} \dot{V}^{2}+\frac{B^{\prime}}{B^{2}}+\frac{D^{\prime}}{D} \Delta\right] & =-\frac{8 T_{D 3}^{2}}{\pi T^{4}} \frac{F(\sigma)}{\sqrt{F^{2}(\sigma)-F^{2}\left(\sigma_{0}\right)}} \sigma^{2} \frac{1}{\cosh ^{2} \alpha} \\
\frac{1}{\sqrt{\Delta}}\left[2 \ddot{V}+2 \frac{B^{\prime}}{B} \dot{V}^{2}+\frac{B^{\prime}}{B^{2}}+\frac{C^{\prime}}{C} \Delta\right] & =-\frac{2 T_{D 3}^{2}}{\pi T^{4}} \frac{F(\sigma)}{F\left(\sigma_{0}\right)} \sigma^{2} \frac{4 \cosh ^{2} \alpha+1}{\cosh ^{4} \alpha} .
\end{aligned}
$$

Assuming $\mathrm{D}=\mathrm{C}$ and with the help of equation (2.12), we solve the above equation and obtain the explicit forms of a, B, C and D:

$$
\begin{aligned}
& a(t)=\exp \left(\int U d t\right) \\
& U=-\frac{8 T_{D 3}^{2}}{\pi T^{4}} \frac{F(\sigma)}{\sqrt{F^{2}(\sigma)-F^{2}\left(\sigma_{0}\right)}} \sigma^{2} \frac{1}{\cosh ^{2} \alpha}+\frac{2 T_{D 3}^{2}}{\pi T^{4}} \frac{F(\sigma)}{F\left(\sigma_{0}\right)} \sigma^{2} \frac{4 \cosh ^{2} \alpha+1}{\cosh ^{4} \alpha} \rightarrow \\
& U=-\frac{8 T_{D 3}^{2}}{\pi T^{4}} \frac{\sigma^{4}}{\sqrt{\sigma^{2}-\sigma_{0}^{2}}}\left(\frac{2 T^{4} \sqrt{1+\frac{\beta^{2}}{\sigma^{4}}}}{3 \sqrt{3}-T^{4} \sqrt{1+\frac{\beta^{2}}{\sigma^{4}}}-\frac{\sqrt{3}}{6} T^{8}\left(1+\frac{\beta^{2}}{\sigma^{4}}\right)}\right)^{2}+ \\
& \frac{2 T_{D 3}^{2}}{\pi T^{4}} \frac{\sigma^{4}}{\sigma_{0}^{2}}\left(4+\left(\frac{2 T^{4} \sqrt{1+\frac{\beta^{2}}{\sigma^{4}}}}{3 \sqrt{3}-T^{4} \sqrt{1+\frac{\beta^{2}}{\sigma^{4}}}-\frac{\sqrt{3}}{6} T^{8}\left(1+\frac{\beta^{2}}{\sigma^{4}}\right)}\right)^{4}\right) \rightarrow \\
& U=-\left[\frac{8 T_{D 3}^{2}\left[\left(\frac{40 T_{F 1} k \pi^{3}}{729}\right)^{2 / 3} t^{2 / 3}+\left(\frac{40 T_{F 1} k \pi^{3}}{729}\right)^{2 / 11} t^{2 / 11}+\ln \left(\frac{16 T_{D 3}\left(T_{F 1} k \pi\right)^{1 / 2}}{81} t\right)\right]}{\pi} \frac{\sigma^{4}}{\sqrt{\sigma^{2}-\sigma_{0}^{2}}} \times\right. \\
& \left.\left(\frac{2\left[\left(\frac{40 T_{F 1} k \pi^{3}}{729}\right)^{2 / 3} t^{2 / 3}+\left(\frac{40 T_{F 1} k \pi^{3}}{729}\right)^{2 / 11} t^{2 / 11}+\ln \left(\frac{16 T_{D 3}\left(T_{F 1} k \pi\right)^{1 / 2}}{81} t\right)\right]^{-4} \sqrt{1+\frac{\beta^{2}}{\sigma^{4}}}}{3 \sqrt{3}-t^{-8 / 3} \sqrt{1+\frac{\beta^{2}}{\sigma^{4}}}-\frac{\sqrt{3}}{6}\left[\left(\frac{40 T_{11} k \pi^{3}}{729}\right)^{2 / 3} t^{2 / 3}+\left(\frac{40 T_{F 1} k \pi^{3}}{729}\right)^{2 / 11} t^{2 / 11}+\ln \left(\frac{16 T_{D 3}\left(T_{F 1} k \pi\right)^{1 / 2}}{81} t\right)\right]^{-8}\left(1+\frac{\beta^{2}}{\sigma^{4}}\right)}\right)^{2}\right] \\
& +\left[\frac{2 T_{D 3}^{2}\left[\left(\frac{40 T_{F 1} k \pi^{3}}{729}\right)^{2 / 3} t^{2 / 3}+\left(\frac{40 T_{F 1} k \pi^{3}}{729}\right)^{2 / 11} t^{2 / 11}+\ln \left(\frac{16 T_{D 3}\left(T_{F 1} k \pi\right)^{1 / 2}}{81} t\right)\right]^{4}}{\pi} \frac{\sigma^{4}}{\sigma_{0}^{2}}(4+\right.
\end{aligned}
$$




$$
\begin{aligned}
& \left.\left(\frac{\left.2\left(\frac{40 T_{F 1} k \pi^{3}}{729}\right)^{2 / 3} t^{2 / 3}+\left(\frac{40 T_{F 1} k \pi^{3}}{729}\right)^{2 / 11} t^{2 / 11}+\ln \left(\frac{16 T_{D 3}\left(T_{F 1} k \pi\right)^{1 / 2}}{81} t\right)\right]^{-4} \sqrt{1+\frac{\beta^{2}}{\sigma^{4}}}}{\left.3 \sqrt{3}-[t]^{-8 / 3} \sqrt{1+\frac{\beta^{2}}{\sigma^{4}}}-\frac{\sqrt{3}}{6}\left(\frac{40 T_{F 1} k \pi^{3}}{729}\right)^{2 / 3} t^{2 / 3}+\left(\frac{40 T_{F 1} k \pi^{3}}{729}\right)^{2 / 11} t^{2 / 11}+\ln \left(\frac{16 T_{D 3}\left(T_{F 1} k \pi\right)^{1 / 2}}{81} t\right)\right]^{-8}\left(1+\frac{\beta^{2}}{\sigma^{4}}\right)}\right)\right] \\
& D=C=\exp \left(\int E d r\right) \\
& E=-\frac{2 T_{D 3}^{2}}{\pi T^{4}} \frac{F(\sigma)}{F\left(\sigma_{0}\right)} \sigma^{2} \frac{4 \cosh ^{2} \alpha+1}{\cosh ^{4} \alpha}+\frac{46 T_{D 3}^{2}}{\pi T^{4}} \frac{F(\sigma)}{\sqrt{F^{2}(\sigma)-F^{2}\left(\sigma_{0}\right)}} \sigma^{2} \frac{1}{\cosh ^{2} \alpha} \rightarrow \\
& E=-\left[\frac{2 T_{D 3}^{2}\left[\left(\frac{40 T_{F 1} k \pi^{3}}{729}\right)^{2 / 3} t^{2 / 3}+\left(\frac{40 T_{F 1} k \pi^{3}}{729}\right)^{2 / 11} t^{2 / 11}+\ln \left(\frac{16 T_{D 3}\left(T_{F 1} k \pi\right)^{1 / 2}}{81} t\right)\right]^{4}}{\pi} \frac{\sigma^{4}}{\sigma_{0}^{2}}(4+\right. \\
& \left.\left(\frac{\left.2\left(\frac{40 T_{F 1} k \pi^{3}}{729}\right)^{2 / 3} t^{2 / 3}+\left(\frac{40 T_{F 1} k \pi^{3}}{729}\right)^{2 / 11} t^{2 / 11}+\ln \left(\frac{16 T_{D 3}\left(T_{F 1} k \pi\right)^{1 / 2}}{81} t\right)\right]^{-4} \sqrt{1+\frac{\beta^{2}}{\sigma^{4}}}}{\left.3 \sqrt{3}-[t]^{-8 / 3} \sqrt{1+\frac{\beta^{2}}{\sigma^{4}}}-\frac{\sqrt{3}}{6}\left(\frac{40 T_{F 1} k \pi^{3}}{729}\right)^{2 / 3} t^{2 / 3}+\left(\frac{40 T_{F 1} k \pi^{3}}{729}\right)^{2 / 11} t^{2 / 11}+\ln \left(\frac{16 T_{D 3}\left(T_{F 1} k \pi\right)^{1 / 2}}{81} t\right)\right]^{-8}\left(1+\frac{\beta^{2}}{\sigma^{4}}\right)}\right)\right] \\
& +\left[\frac{48 T_{D 3}^{2}\left[\left(\frac{40 T_{F 1} k \pi^{3}}{729}\right)^{2 / 3} t^{2 / 3}+\left(\frac{40 T_{F 1} k \pi^{3}}{729}\right)^{2 / 11} t^{2 / 11}+\ln \left(\frac{16 T_{D 3}\left(T_{F 1} k \pi\right)^{1 / 2}}{81} t\right)\right]^{4}}{\pi} \frac{\sigma^{4}}{\sqrt{\sigma^{2}-\sigma_{0}^{2}}} \times\right. \\
& \left.\left(\frac{2\left[\left(\frac{40 T_{F 1} k \pi^{3}}{729}\right)^{2 / 3} t^{2 / 3}+\left(\frac{40 T_{F 1} k \pi^{3}}{729}\right)^{2 / 11} t^{2 / 11}+\ln \left(\frac{16 T_{D 3}\left(T_{F 1} k \pi\right)^{1 / 2}}{81} t\right)\right]^{-4} \sqrt{1+\frac{\beta^{2}}{\sigma^{4}}}}{3 \sqrt{3}-t^{-8 / 3} \sqrt{1+\frac{\beta^{2}}{\sigma^{4}}}-\frac{\sqrt{3}}{6}\left[\left(\frac{40 T_{F 1} k \pi^{3}}{729}\right)^{2 / 3} t^{2 / 3}+\left(\frac{40 T_{F 1} k \pi^{3}}{729}\right)^{2 / 11} t^{2 / 11}+\ln \left(\frac{16 T_{D 3}\left(T_{F 1} k \pi\right)^{1 / 2}}{81} t\right)\right]^{-8}\left(1+\frac{\beta^{2}}{\sigma^{4}}\right)}\right)^{2}\right] \\
& \rightarrow E \simeq-U \\
& B=\exp \left(\int w d r\right) \\
& w=-\left(\frac{1}{1+2 \dot{P}^{2}}\right)\left(\frac{2 T_{D 3}^{2}}{\pi T^{4}} \frac{F(\sigma)}{F\left(\sigma_{0}\right)} \sigma^{2} \frac{4 \cosh ^{2} \alpha+1}{\cosh ^{4} \alpha}\right)-2 \ddot{U} \rightarrow \\
& w=-2 \ddot{U}-\left[\frac{2 T_{D 3}^{2}\left[\left(\frac{40 T_{F 1} k \pi^{3}}{729}\right)^{2 / 3} t^{2 / 3}+\left(\frac{40 T_{F 1} k \pi^{3}}{729}\right)^{2 / 11} t^{2 / 11}+\ln \left(\frac{16 T_{D 3}\left(T_{F 1} k \pi\right)^{1 / 2}}{81} t\right)\right]^{4}}{\pi} \frac{\sigma^{4}}{\sigma_{0}^{2}}(4+\right. \\
& \left.\left.\left(\frac{\left.2\left(\frac{40 T_{F 1} k \pi^{3}}{729}\right)^{2 / 3} t^{2 / 3}+\left(\frac{40 T_{F 1} k \pi^{3}}{729}\right)^{2 / 11} t^{2 / 11}+\ln \left(\frac{16 T_{D 3}\left(T_{F 1} k \pi\right)^{1 / 2}}{81} t\right)\right]^{-4} \sqrt{1+\frac{\beta^{2}}{\sigma^{4}}}}{\left.3 \sqrt{3}-[t]^{-8 / 3} \sqrt{1+\frac{\beta^{2}}{\sigma^{4}}}-\frac{\sqrt{3}}{6}\left(\frac{40 T_{F 1} k \pi^{3}}{729}\right)^{2 / 3} t^{2 / 3}+\left(\frac{40 T_{F 1} k \pi^{3}}{729}\right)^{2 / 11} t^{2 / 11}+\ln \left(\frac{16 T_{D 3}\left(T_{F 1} k \pi\right)^{1 / 2}}{81} t\right)\right]^{-8}\left(1+\frac{\beta^{2}}{\sigma^{4}}\right)}\right)^{4}\right)\right] \\
& \rightarrow w \simeq-U \text {. }
\end{aligned}
$$

This equation indicates that while the temperature is decreased, the parameters of wormhole are reduced to lower values and tended to zero at $\sigma_{0}=V=0$ and $t=t_{\text {end }}$ (see figures 1 and 2). This means that the wormhole is disappeared at the end of inflation, however the scale factor of universe is increased very fast and tended to large values in this epoch (see figure 3). 


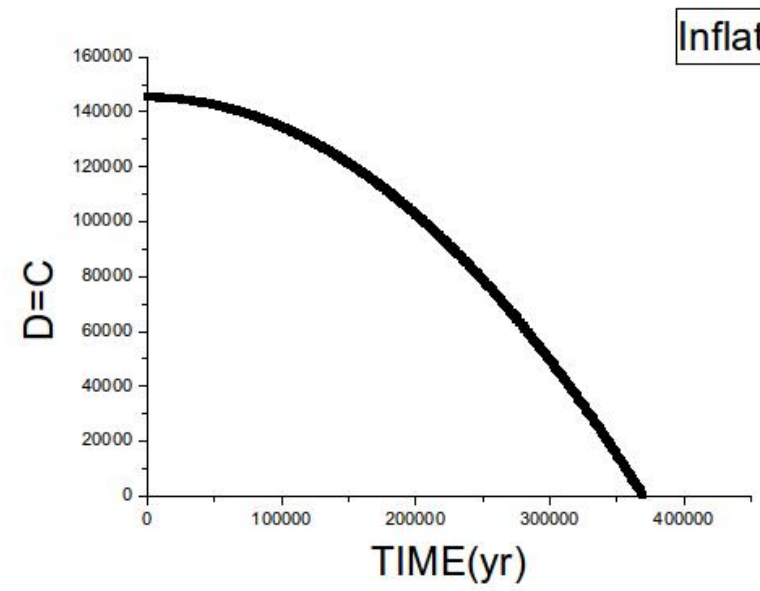

Figure 1. The wormhole parameter $(\mathrm{D}=\mathrm{C})$ for inflation era of expansion history as a function of $\mathrm{t}$ where $\mathrm{t}$ is the age of universe. In this plot, we choose $t_{\mathrm{birth}}=0, t_{\mathrm{end}}=380000, T_{\mathrm{birth}}=10^{32}$ and $T_{\text {end }}=10^{9}$.

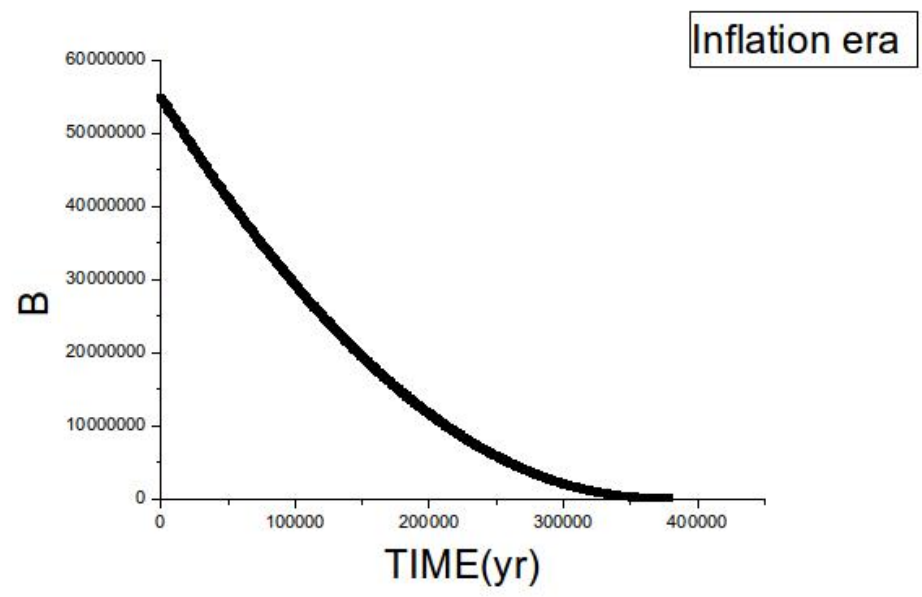

Figure 2. The wormhole parameter (B) for inflation era of expansion history as a function of $\mathrm{t}$ where $\mathrm{t}$ is age of universe. In this plot, we choose $t_{\mathrm{birth}}=0, t_{\text {end }}=380000, T_{\text {birth }}=10^{32}$ and $T_{\text {end }}=10^{9}$.

\section{The birth and death of cylindrical wormhole during late time acceleration}

In this section, we discuss that with decreasing the separation distance between braneantibrane, a tachyon is born, grows very fast and causes formation of a new cylindrical wormhole. This wormhole transfers energy from extra dimensions into our universe according to which the second phase of acceleration takes place.

To construct a non-phantom model, we consider a set of D3- $\bar{D} 3$-brane pairs in the background (2.7) which are placed at points $z_{1}=l / 2$ and $z_{2}=-l / 2$ respectively so that the separation between the brane and antibrane is 1 . For the simple case of a single 


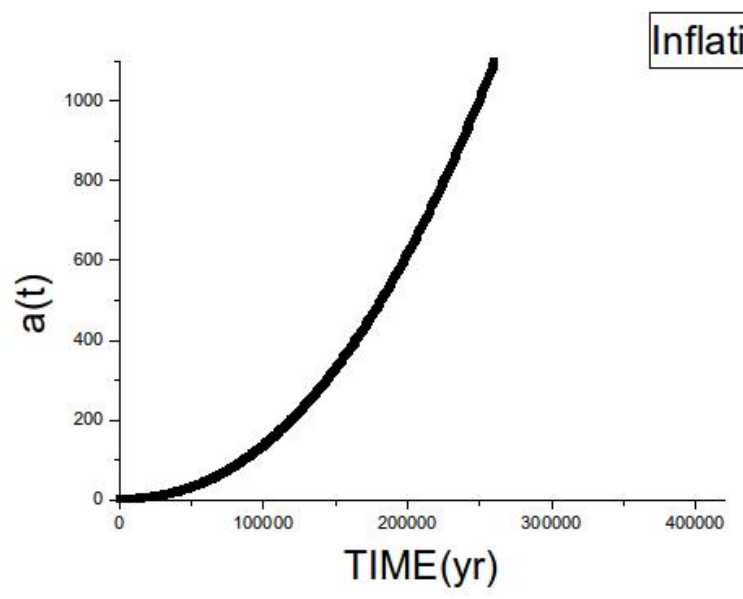

Figure 3. The scale factor (a) for inflation era of expansion history as a function of $t$ where $t$ is age of universe. In this plot, we choose $t_{\text {birth }}=0, t_{\text {end }}=380000, T_{\text {birth }}=10^{32}$ and $T_{\text {end }}=10^{9}$.

D3- $\bar{D} 3$-brane pair with an open string tachyon, the action is [19-24]:

$$
\begin{aligned}
S= & -\tau_{3} \int d^{9} \sigma \sum_{i=1}^{2} V(T A, l) e^{-\phi}\left(\sqrt{-\operatorname{det} A_{i}}\right) \\
\left(A_{i}\right)_{a b}= & \left(g_{M N}-\frac{T A^{2} l^{2}}{Q} g_{M z} g_{z N}\right) \partial_{a} x_{i}^{M} \partial_{b} x_{i}^{M}+F_{a b}^{i}+\frac{1}{2 Q}\left(\left(D_{a} T A\right)\left(D_{b} T A\right)^{*}+\left(D_{a} T A\right)^{*}\left(D_{b} T A\right)\right) \\
& +i l\left(g_{a z}+\partial_{a} z_{i} g_{z z}\right)\left(T A\left(D_{b} T A\right)^{*}-T A^{*}\left(D_{b} T A\right)\right)+i l\left(T A\left(D_{a} T A\right)^{*}-T A^{*}\left(D_{a} T A\right)\right) \times \\
& \left(g_{b z}+\partial_{b} z_{i} g_{z z}\right)\left(1-\frac{\pi^{2} N T^{4}}{6 T_{D 3}}\right),
\end{aligned}
$$

where

$$
\begin{aligned}
Q & =1+T A^{2} l^{2} g_{z z}, \\
D_{a} T A & =\partial_{a} T A-i\left(A_{2, a}-A_{1, a}\right) T A, V(T A, l)=g_{s} V(T A) \sqrt{Q}, \\
e^{\phi} & =g_{s}\left(1+\frac{R^{4}}{z^{4}}\right)^{-\frac{1}{2}}
\end{aligned}
$$

$\phi, A_{2, a}$ and $F_{a b}^{i}$ are dilaton field, the gauge field and field strength on the world-volume of the non-BPS brane respectively, TA is the tachyon field, $\tau_{3}$ is the brane tension and $\mathrm{V}$ (TA) is the tachyon potential. The indices a,b denote the tangent directions of D-branes, while the indices $\mathrm{M}, \mathrm{N}$ run over the background of ten-dimensional space-time directions. The Dp-brane and the anti-Dp-brane are labeled by $i=1$ and 2 respectively. Then the separation between these D-branes is defined by $z_{2}-z_{1}=l$. Also, in writing the above equations we are using the convention $2 \pi \dot{\alpha}=1$. A potential which has been used in most papers is [25-27]:

$$
V(T A)=\frac{\tau_{3}}{\cosh \sqrt{\pi} T A} .
$$

Let us consider the only $\sigma$ dependence of the tachyon field TA for simplicity and set the gauge fields to zero. In this case, the action (3.1) in the region $r>R$ and $T A^{\prime} \sim$ constant 
simplifies to

$$
L \simeq-\frac{\tau_{3}}{g_{s}} \int d \sigma \sigma^{2} V(T A)\left(\sqrt{D_{1, T A}}+\sqrt{D_{2, T A}}\right)\left(1-\frac{\pi^{2} N T^{4}}{6 T_{D 3}}\right)
$$

where

$$
D_{1, T A}=D_{2, T A} \equiv D_{T A}=1+\frac{l^{\prime}(\sigma)^{2}}{4}+T A^{2}-T A^{\prime 2}
$$

we assume that $T A l \ll T A^{\prime}$. Now, we study the Hamiltonian corresponding to the above Lagrangian. To derive this we need the canonical momentum density $\Pi=\frac{\partial L}{\partial T A}$ associated with the tachyon:

$$
\Pi=\frac{V(T A) \dot{T A}}{\sqrt{1+\frac{l^{\prime}(\sigma)^{2}}{4}+\dot{T A^{2}}-T A^{\prime 2}}}\left(1-\frac{\pi^{2} N T^{4}}{6 T_{D 3}}\right)
$$

so that the Hamiltonian can be obtained as:

$$
H_{\mathrm{DBI}}=4 \pi \int d \sigma \sigma^{2} \Pi \dot{T} A-L .
$$

By choosing $\dot{T A}=2 T A^{\prime}$, this gives:

$$
H_{\mathrm{DBI}}=4 \pi \int d \sigma \sigma^{2}\left[\Pi\left(\dot{T A}-\frac{1}{2} T A^{\prime}\right)\right]+\frac{1}{2} T A \partial_{\sigma}\left(\Pi \sigma^{2}\right)-L .
$$

In this equation, we have in the second step integration by parts the term proportional to $\dot{T A}$, indicating that a tachyon can be studied as a Lagrange multiplier imposing the constraint $\partial_{\sigma}\left(\Pi \sigma^{2} V(T A)\right)=0$ on the canonical momentum. Solving this equation yields:

$$
\Pi=\frac{\beta}{4 \pi \sigma^{2}}
$$

where $\beta$ is a constant. Using (3.9) in (3.8), we get:

$$
\begin{aligned}
& H_{\mathrm{DBI}}=\int d \sigma V(T A) \sqrt{1+\frac{l^{\prime}(\sigma)^{2}}{4}+\dot{T A^{2}}-T A^{\prime 2}} F_{\mathrm{DBI}}, \\
& F_{\mathrm{DBI}}=\sigma^{2} \sqrt{1+\frac{\beta}{\sigma^{2}}}\left(1-\frac{\pi^{2} N T^{4}}{6 T_{D 3}}\right) .
\end{aligned}
$$

The output of EOM for $l(\sigma)$, calculated by varying (3.10), is

$$
\left(\frac{l^{\prime} F_{\mathrm{DBI}}}{4 \sqrt{1+\frac{l^{\prime}(\sigma)^{2}}{4}}}\right)^{\prime}=0 .
$$

Solving this equation, we obtain:

$$
(\sigma)=2\left(\frac{l_{0}}{2}-\int_{\sigma}^{\infty} d \sigma\left(\frac{F_{\mathrm{DBI}}(\sigma)}{F_{\mathrm{DBI}}\left(\sigma_{0}\right)}-1\right)^{-\frac{1}{2}}\right) .
$$


This solution, for non-zero $\sigma_{0}$ represents a wormhole with a finite size throat. This equation indicates that the separation distance between two branes is $l_{0}$ at the birth of wormhole $\left(\sigma_{0}\right)$, decreases with time and shrinks to zero at larger values of throat. On the hand, to obtain the explicit form of a tachyon, we are using the equation of motion extracted from action (3.4):

$$
\left(\frac{1}{\sqrt{D_{T A}}} T A^{\prime}(\sigma)\right)^{\prime}=\frac{1}{\sqrt{D_{T A}}}\left[\frac{\left(V(T A) F_{\mathrm{DBI}}\right)}{F_{\mathrm{DBI}} V(T A)^{\prime}}\left(D_{T A}-T A^{\prime}(\sigma)^{2}\right)\right]
$$

with a solution

$$
T A \sim \sqrt{\frac{\sigma_{0}^{2}}{\sigma_{0}^{2}-\sigma^{2}}}\left(\frac{1}{1+\frac{\pi^{2} N T^{4}}{6 T_{D 3}}}\right) .
$$

This equation shows that a tachyon is zero before the birth of a wormhole $\left(\sigma_{0}=0\right)$ and with a decrease in temperature and an increase in the throat of a wormhole it grows to larger values.

At this stage, we consider the late time acceleration of universe in thermal BIon. To achieve this, we calculate the contribution of a tachyonic wormhole to the four-dimensional universe energy momentum tensor. We have:

$$
\begin{aligned}
T^{00} & =V(T A) F_{\mathrm{DBI}} \sqrt{D_{T A}}, \\
T^{44} & =-V(T A) F_{\mathrm{DBI}} \frac{1}{\sqrt{D_{T A}}}\left(T A^{2} l^{2}+\frac{i^{2}}{4}\right) \\
T^{i i} & =-V(T A) F_{\mathrm{DBI}} \frac{Q}{\sqrt{D_{T A}}}, i=1,2,3 .
\end{aligned}
$$

Setting the energy momentum tensor of equations (2.22) and (3.15) in the conservation law of equations (2.21) and (2.24) and employing (2.23) yields:

$$
\begin{aligned}
6 \frac{\dot{a}^{2}}{a^{2}}+\left(\frac{D^{\prime}}{D}+\frac{C^{\prime}}{C}\right) \sqrt{\Delta} & =V(T A) F_{\mathrm{DBI}} \sqrt{D_{T A}} \\
4 \frac{\ddot{a}}{a}+2 \frac{\dot{a}^{2}}{a^{2}}+\frac{1}{\sqrt{\Delta}}\left[2 \ddot{V}+2 \frac{B^{\prime}}{B} \dot{V}^{2}+\frac{B^{\prime}}{B^{2}}+\frac{D^{\prime}}{D} \Delta\right] & =-V(T A) F_{\mathrm{DBI}} \frac{Q}{\sqrt{D_{T A}}} \\
\frac{1}{\sqrt{\Delta}}\left[2 \ddot{V}+2 \frac{B^{\prime}}{B} \dot{V}^{2}+\frac{B^{\prime}}{B^{2}}+\frac{C^{\prime}}{C} \Delta\right] & =-V(T A) F_{\mathrm{DBI}} \frac{1}{\sqrt{D_{T A}}}\left(T A^{2} l^{2}+\frac{\dot{l}^{2}}{4}\right) .
\end{aligned}
$$

Solving these equations simultaneously, assuming $(\mathrm{D}=\mathrm{C}), V(t)=\exp \left(-\int P_{0} d t\right)$ and using equations (2.12), (3.12) and (3.14), we obtain the explicit form of wormhole parameters and the scale factor of universe:

$$
\begin{aligned}
& a(t)=\exp \left(\int d t G(t)\right) \\
& G(t)=\exp \left(\frac{6 T_{D 3}\left[\left(\frac{40 T_{F 1} k \pi^{3}}{729}\right)^{2 / 3} t^{2 / 3}+\left(\frac{40 T_{F 1} k \pi^{3}}{729}\right)^{2 / 11} t^{2 / 11}+\ln \left(\frac{16 T_{D 3}\left(T_{F 1} k \pi\right)^{1 / 2}}{81} t\right)\right]^{4}}{\pi^{2} N}\right) \times
\end{aligned}
$$




$$
\begin{aligned}
& \left(\frac{6 T_{D 3}\left[\left(\frac{40 T_{F 1} k \pi^{3}}{729}\right)^{2 / 3} t^{2 / 3}+\left(\frac{40 T_{F 1} k \pi^{3}}{729}\right)^{2 / 11} t^{2 / 11}+\ln \left(\frac{16 T_{D 3}\left(T_{F 1} k \pi\right)^{1 / 2}}{81} t\right)\right]}{\pi^{2} N}\right) \sqrt{\frac{\sigma_{0}^{2}}{\sigma_{0}^{2}-\sigma^{2}}} \\
& D=C=\exp \left(\int H d r\right) \\
& H=\sqrt{1+\left(\frac{\sigma_{0}^{2}}{\sigma_{0}^{2}-\sigma^{2}}\right)\left(1+\frac{\pi^{2} N\left[\left(\frac{40 T_{F 1} k \pi^{3}}{729}\right)^{2 / 3} t^{2 / 3}+\left(\frac{40 T_{F 1} k \pi^{3}}{729}\right)^{2 / 11} t^{2 / 11}+\ln \left(\frac{16 T_{D 3}\left(T_{F 1} k \pi\right)^{1 / 2}}{81} t\right)\right]^{4}}{6 T_{D 3}}\right)^{2}} \times \\
& \exp \left(\frac{6 T_{D 3}\left[\left(\frac{40 T_{F 1} k \pi^{3}}{729}\right)^{2 / 3} t^{2 / 3}+\left(\frac{40 T_{F 1} k \pi^{3}}{729}\right)^{2 / 11} t^{2 / 11}+\ln \left(\frac{16 T_{D 3}\left(T_{F 1} k \pi\right)^{1 / 2}}{81} t\right)\right]^{4}}{\pi^{2} N}\right) \times \\
& \left(\frac{6 T_{D 3}\left[\left(\frac{40 T_{F 1} k \pi^{3}}{729}\right)^{2 / 3} t^{2 / 3}+\left(\frac{40 T_{F 1} k \pi^{3}}{729}\right)^{2 / 11} t^{2 / 11}+\ln \left(\frac{16 T_{D 3}\left(T_{F 1} k \pi\right)^{1 / 2}}{81} t\right)\right]^{4}}{\pi^{2} N}\right) \sigma^{2} \sqrt{1+\frac{\beta}{\sigma^{2}}} \\
& B=\exp \left(\int X d r\right) \\
& X=\frac{\left[\left(\frac{40 T_{F 1} k \pi^{3}}{729}\right)^{2 / 3} t^{2 / 3}+\left(\frac{40 T_{F 1} k \pi^{3}}{729}\right)^{2 / 11} t^{2 / 11}+\ln \left(\frac{16 T_{D 3}\left(T_{F 1} k \pi\right)^{1 / 2}}{81} t\right)\right]^{2} 4\left(\frac{l_{0}}{2}-\int_{\sigma}^{\infty} d \sigma\left(\frac{\sigma^{2}}{\sigma_{0}^{2}}-1\right)^{-1 / 2}\right)^{2}}{\sqrt{\left(\frac{\sigma_{0}^{2}}{\sigma_{0}^{2}-\sigma^{2}}\right)+\left[\left(\frac{40 T_{F 1} k \pi^{3}}{729}\right)^{2 / 3} t^{2 / 3}+\left(\frac{40 T_{F 1} k \pi^{3}}{729}\right)^{2 / 11} t^{2 / 11}+\ln \left(\frac{16 T_{D 3}\left(T_{F 1} k \pi\right)^{1 / 2}}{81} t\right)\right]^{2}}} \\
& +\frac{\frac{\sigma_{0}^{2}}{\sigma_{0}^{2}-\sigma^{2}}}{\sqrt{\left(\frac{\sigma_{0}^{2}}{\sigma_{0}^{2}-\sigma^{2}}\right)+\left[\left(\frac{40 T_{F 1} k \pi^{3}}{729}\right)^{2 / 3} t^{2 / 3}+\left(\frac{40 T_{F 1} k \pi^{3}}{729}\right)^{2 / 11} t^{2 / 11}+\ln \left(\frac{16 T_{D 3}\left(T_{F 1} k \pi\right)^{1 / 2}}{81} t\right)\right]^{2}}} \times \\
& \exp \left(\frac{6 T_{D 3}\left[\left(\frac{40 T_{F 1} k \pi^{3}}{729}\right)^{2 / 3} t^{2 / 3}+\left(\frac{40 T_{F 1} k \pi^{3}}{729}\right)^{2 / 11} t^{2 / 11}+\ln \left(\frac{16 T_{D 3}\left(T_{F 1} k \pi\right)^{1 / 2}}{81} t\right)\right]^{4}}{\pi^{2} N}\right) \times \\
& \left(\frac{6 T_{D 3}\left[\left(\frac{40 T_{F 1} k \pi^{3}}{729}\right)^{2 / 3} t^{2 / 3}+\left(\frac{40 T_{F 1} k \pi^{3}}{729}\right)^{2 / 11} t^{2 / 11}+\ln \left(\frac{16 T_{D 3}\left(T_{F 1} k \pi\right)^{1 / 2}}{81} t\right)\right]^{4}}{\pi^{2} N}\right) \sigma^{2} \sqrt{1+\frac{\beta}{\sigma^{2}}} .
\end{aligned}
$$

These solutions indicate that the wormhole parameters and also the scale factor are increased with time and shrink to infinity at $\left(\sigma=\sigma_{0}, t=t_{\text {rip }}\right)$. In figures 4,5 and 6 , we compare wormhole parameters and the scale factor. As can be seen from these figures, the value of scale factor is higher than the corresponding values for the wormhole parameters at the beginning of acceleration era. On the other hand, the rate of growth for the wormhole parameters is more considerable. For this reason, we predict that these parameters overtake the scale factor and lead to the destruction of universe at future big rip singularity [28]. 


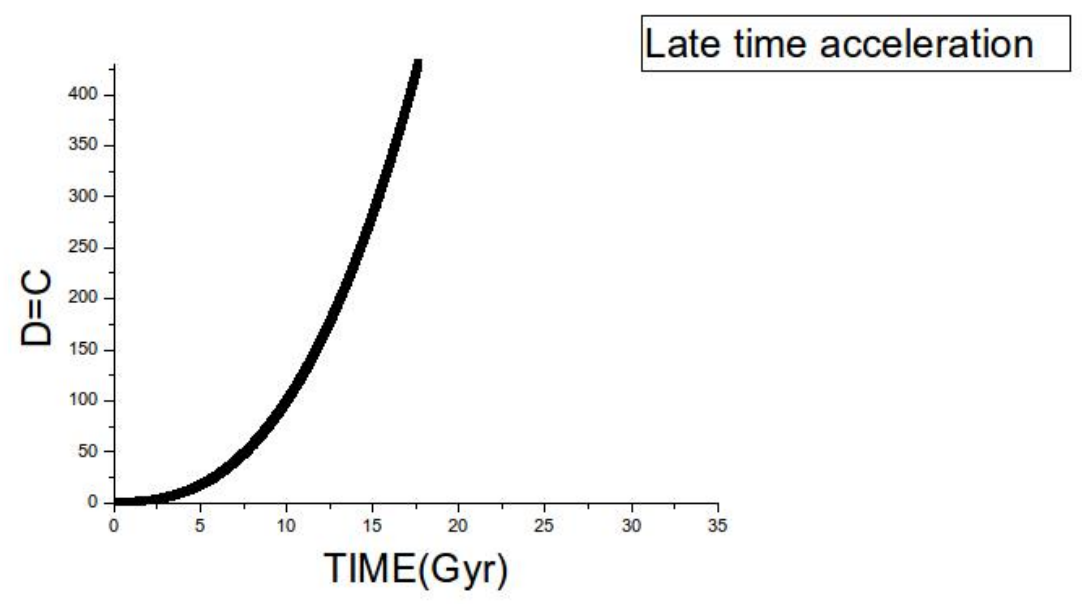

Figure 4. The wormhole parameter $(\mathrm{D}=\mathrm{C})$ for late time acceleration era of expansion history as a function of $\mathrm{t}$ where $\mathrm{t}$ is age of universe. In this plot, we choose $t_{\text {late }}=.4 \mathrm{Gyr}, t_{\text {rip }}=33 \mathrm{Gyr}$, $T_{\text {late }}=10^{4}$ and $T_{\text {end }}=0$.

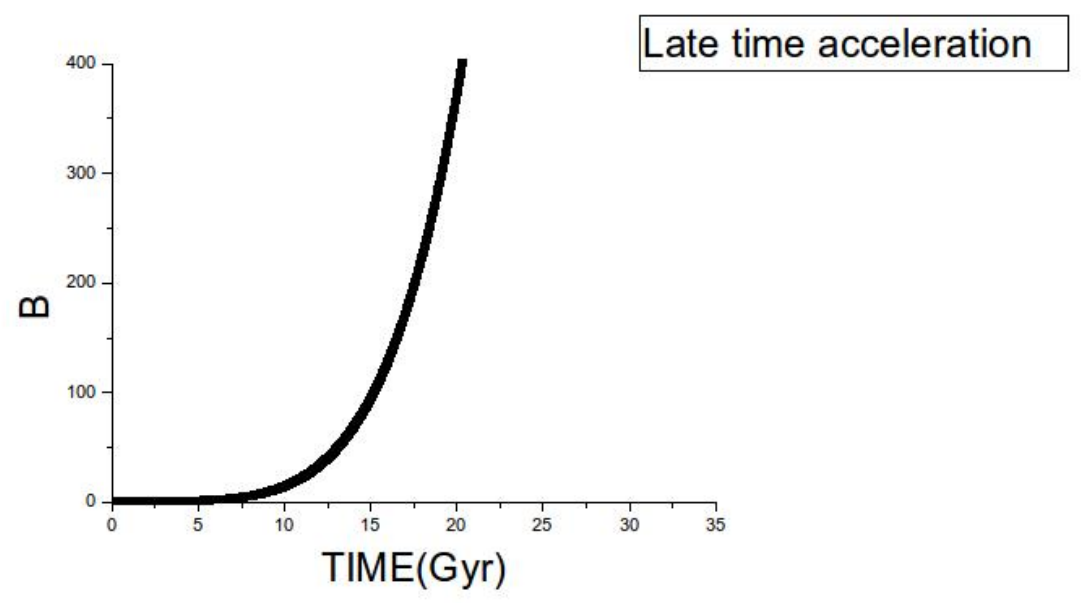

Figure 5. The wormhole parameter (B) for late time acceleration era of expansion history as a function of $\mathrm{t}$ where $\mathrm{t}$ is age of universe. In this plot, we choose $t_{\text {late }}=.4 \mathrm{Gyr}, t_{\text {rip }}=33 \mathrm{Gyr}$, $T_{\text {late }}=10^{4}$ and $T_{\text {end }}=0$.

\section{Summary and discussion}

Recently, the stability analysis of cylindrical thin shell wormholes has been studied in the literature. In this paper, we have proposed a new model that allows us to account for dynamics of this wormhole during different epochs of cosmic history from inflation to recent observed acceleration era. In this model, coincided with the birth of universe at the corresponding point, the early wormhole is born. At this point, $k$ black fundamental strings are transited to BIon which is a configuration of a universe brane and a universe anti-brane connected by a wormhole. This wormhole transfers energy from another universe to our own universe and causes inflation. We have shown that two universe-branes can be connected by an unstable cylindrical thin shell wormhole that vanishes very fast. After 


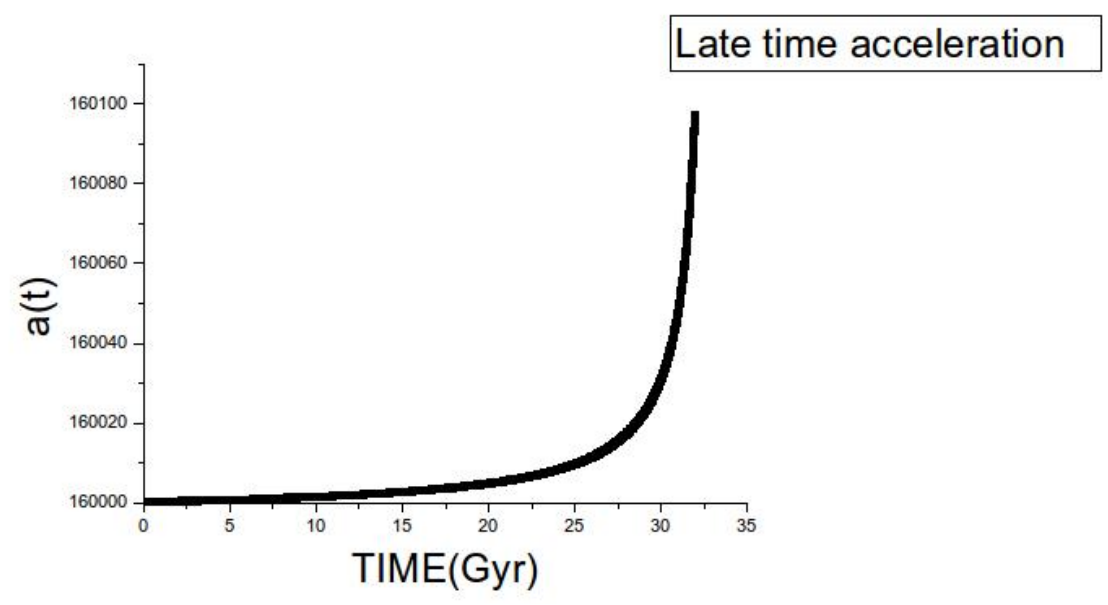

Figure 6. The scale factor (a) for late time acceleration era of expansion history as a function of $\mathrm{t}$ where $\mathrm{t}$ is age of universe. In this plot, we choose $t_{\text {late }}=.4 \mathrm{Gyr}, t_{\text {rip }}=33 \mathrm{Gyr}, T_{\text {late }}=10^{4}$ and $T_{\text {end }}=0$.

the wormhole death, there isn't any channel for flowing energy into our universe brane, inflation ends and a non phantom era begins. With decreasing the separation between universe branes, the second type of cylindrical thin shell wormholes, named as tachyonic wormholes are created. In this condition, two universe branes are connected again and late time acceleration era is started. After that we have considered the stability of these wormholes and came to a conclusion that they will vanish at a future singularity.

\section{Acknowledgments}

Authors wish to thank Professor S. Habib Mazharimousavi and Professor M. Halilsoy for their nice comments that help us to improve our paper.

Open Access. This article is distributed under the terms of the Creative Commons Attribution License (CC-BY 4.0), which permits any use, distribution and reproduction in any medium, provided the original author(s) and source are credited.

\section{References}

[1] A. Einstein and N. Rosen, The Particle Problem in the General Theory of Relativity, Phys. Rev. 48 (1935) 73 [inSPIRE].

[2] M.S. Morris and K.S. Thorne, Wormholes in space-time and their use for interstellar travel: A tool for teaching general relativity, Am. J. Phys. 56 (1988) 395 [InSPIRE].

[3] M. Visser, Traversable wormholes: Some simple examples, Phys. Rev. D 39 (1989) 3182 [arXiv:0809.0907] [INSPIRE].

[4] M. Visser, Traversable wormholes from surgically modified Schwarzschild space-times, Nucl. Phys. B 328 (1989) 203 [arXiv:0809.0927] [INSPIRE].

[5] M. Visser, Lorentzian Wormholes - from Einstein to Hawking, American Institute of Physics, New York, U.S.A. (1995). 
[6] G. Grignani, T. Harmark, A. Marini, N.A. Obers and M. Orselli, Heating up the BIon, JHEP 06 (2011) 058 [arXiv: 1012.1494] [INSPIRE].

[7] G. Grignani, T. Harmark, A. Marini, N.A. Obers and M. Orselli, Thermodynamics of the hot BIon, Nucl. Phys. B 851 (2011) 462 [arXiv:1101.1297] [INSPIRE].

[8] M.R. Setare and A. Sepehri, Constructing warm inflationary model in finite temperature BIon, arXiv: 1410.2552 [INSPIRE].

[9] M.R. Setare and A. Sepehri, Constructing warm inflationary model in finite temperature BIon, arXiv: 1410.2552 [INSPIRE].

[10] A. Sepehri, F. Rahaman, A. Pradhan and I.H. Sardar, Emergence and Expansion of Cosmic Space in BIonic system, Phys. Lett. B 741 (2015) 92 [arXiv:1501.00428] [INSPIRE].

[11] E.F. Eiroa and C. Simeone, Cylindrical thin shell wormholes, Phys. Rev. D 70 (2004) 044008 [gr-qc/0404050] [INSPIRE].

[12] E.F. Eiroa and C. Simeone, Some general aspects of thin-shell wormholes with cylindrical symmetry, Phys. Rev. D 81 (2010) 084022 [arXiv:0912.5496] [INSPIRE].

[13] E.F. Eiroa and C. Simeone, Brans-Dicke cylindrical wormholes, Phys. Rev. D 82 (2010) 084039 [arXiv: 1008.0382] [INSPIRE].

[14] M.G. Richarte, Cylindrical Wormholes with Positive Cosmological Constant, Phys. Rev. D 88 (2013) 027507 [arXiv: 1308.2651] [INSPIRE].

[15] K.A. Bronnikov and J.P.S. Lemos, Cylindrical wormholes, Phys. Rev. D 79 (2009) 104019 [arXiv: 0902.2360] [INSPIRE].

[16] N.M. Garcia, F.S.N. Lobo and M. Visser, Generic spherically symmetric dynamic thin-shell traversable wormholes in standard general relativity, Phys. Rev. D 86 (2012) 044026 [arXiv: 1112.2057] [INSPIRE].

[17] E. Rubín de Celis, O.P. Santillan and C. Simeone, Probing global aspects of a geometry by the self-force on a charge: cylindical thin-shell wormholes, Phys. Rev. D 86 (2012) 124009 [arXiv: 1210.4149] [INSPIRE].

[18] S.H. Mazharimousavi, M. Halilsoy and Z. Amirabi, Stability of generic cylindrical thin shell wormholes, Phys. Rev. D 89 (2014) 084003 [arXiv: 1403.2861] [INSPIRE].

[19] G. Grignani, T. Harmark, A. Marini and M. Orselli, Thermal DBI action for the D3-brane at weak and strong coupling, JHEP 03 (2014) 114 [arXiv:1311.3834] [INSPIRE].

[20] A. Sen, Dirac- Born-Infeld action on the tachyon kink and vortex, Phys. Rev. D 68 (2003) 066008 [hep-th/0303057] [INSPIRE].

[21] M.R. Garousi, D-brane $\bar{D}$-brane effective action and brane interaction in open string channel, JHEP 01 (2005) 029 [hep-th/0411222] [INSPIRE].

[22] A. Dhar and P. Nag, Sakai-Sugimoto model, Tachyon Condensation and Chiral symmetry Breaking, JHEP 01 (2008) 055 [arXiv: 0708.3233] [INSPIRE].

[23] A. Dhar and P. Nag, Tachyon condensation and quark mass in modified Sakai-Sugimoto model, Phys. Rev. D 78 (2008) 066021 [arXiv:0804.4807] [INSPIRE].

[24] M.R. Setare, A. Sepehri and V. Kamali, Constructing warm inflationary model in brane-antibrane system, Phys. Lett. B 735 (2014) 84 [arXiv:1405.7949] [INSPIRE]. 
[25] C.-j. Kim, H.B. Kim, Y.-b. Kim and O.-K. Kwon, Electromagnetic string fluid in rolling tachyon, JHEP 03 (2003) 008 [hep-th/0301076] [INSPIRE].

[26] F. Leblond and A.W. Peet, SD brane gravity fields and rolling tachyons, JHEP 04 (2003) 048 [hep-th/0303035] [INSPIRE].

[27] N.D. Lambert, H. Liu and J.M. Maldacena, Closed strings from decaying D-branes, JHEP 03 (2007) 014 [hep-th/0303139] [INSPIRE].

[28] R.R. Caldwell, M. Kamionkowski and N.N. Weinberg, Phantom energy and cosmic doomsday, Phys. Rev. Lett. 91 (2003) 071301 [astro-ph/0302506] [INSPIRE]. 